\title{
Capillary-gravity waves on a dielectric fluid of finite depth under normal electric field
}

\author{
Tao Gao ${ }^{\mathrm{a}}$, Alex Doak ${ }^{\mathrm{b}}$, Jean-Marc Vanden-Broeck ${ }^{\mathrm{b}}$, Zhan Wang c,d,*

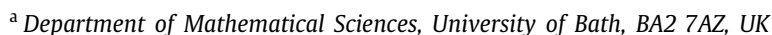 \\ ${ }^{\mathrm{b}}$ Department of Mathematics, University College London, WC1E 6BT, UK \\ ${ }^{\mathrm{c}}$ Institute of Mechanics, Chinese Academy of Sciences, Beijing 100190, China \\ ${ }^{\mathrm{d}}$ School of Engineering Sciences, University of Chinese Academy of Sciences, Beijing 100049, China
}

\section{A R T I C L E I N F O}

\section{Article history:}

Received 27 October 2018

Received in revised form 30 March 2019

Accepted 15 April 2019

Available online 20 June 2019

\section{Keywords:}

Surface wave

Solitary wave

Electrohydrodynamics

Capillary wave

\begin{abstract}
A B S T R A C T
In this work we consider two-dimensional capillary-gravity waves propagating under the influence of a vertical electric field on a dielectric of finite depth bounded above by a perfectly conducting and hydrodynamically passive fluid. Both linear and weakly nonlinear theories are developed, and long-wave model equations are derived based on the analyticity of the Dirichlet-Neumann operator. Fully nonlinear computations are carried out by using a time-dependent conformal mapping method. Solitary waves are found, and their stability characteristics subject to longitudinal perturbations are studied numerically. The shedding of stable solitary waves is achieved by moving a Gaussian pressure on the free surface with the speed close to a phase speed minimum and removing the pressure after a period of time. The novel result shows that a depression bright solitary wave and an elevation generalized solitary wave co-exist in the solitary-wave excitation.
\end{abstract}

(c) 2019 Elsevier Masson SAS. All rights reserved.

\section{Introduction}

Electrohydrodynamics (EHD), which is concerned with the coupling between electric fields and fluid flows, enjoys a wide range of applications in chemistry and engineering, such as coating processes in [1], and cooling systems in a conducting pump in [2]. In practice, an EHD problem usually involves a free surface or an interface between two liquids. Therefore, a good understanding of wave motions under electric fields benefits the engineering community. Research on EHD interfacial waves was first conducted by Taylor \& McEwan in [3]. Their theoretical and experimental results showed that normal electric fields can lead to a destabilization of the interface between a conducting fluid and a dielectric. A few years later, Melcher \& Schwarz performed a linear stability analysis of the problem under tangential electric fields, which were shown to be capable of regularizing short waves in [4]. These two early works described the effect of electric fields on the linear stability of interfacial waves. The study was then extended to many other EHD problems. For example, two works by $[5,6]$ showed the control and suppression of the Rayleigh-Taylor instability using horizontal electric fields. Nonlinear EHD Kelvin-Helmholtz instability was investigated in [7, 8]. Large amplitude travelling waves in electrified fluid sheets

\footnotetext{
* Corresponding author at: Institute of Mechanics, Chinese Academy of Sciences, Beijing 100190, China

E-mail address: zwang@imech.ac.cn (Z. Wang).
}

were computed using the full Euler equations in [9]. The touchdown singularity was observed in a thin film in [10], where the lowest point of the fluid interface reaches the solid bottom. The theoretical studies in the aforementioned references employed the method of multiple scales, whereas the numerical results were obtained either by a boundary integral method or direct numerical simulations.

In the absence of electric fields, the problem reduces to the study of classic capillary-gravity waves. It is well acknowledged that a Korteweg-de Vries ( $\mathrm{KdV}$ ) equation can be derived for twodimensional long capillary-gravity waves. The equation admits elevation solitons for $\tau>1 / 3$, and depression solitons for $\tau<$ $1 / 3$, where $\tau$ is the Bond number defined by

$\tau=\frac{\sigma}{\rho g h^{2}}$,

with $\sigma$ being the coefficient of surface tension, $g$ the acceleration due to gravity, $\rho$ the fluid density and $h$ the thickness of the fluid layer. However, when solving the full Euler equations, depression and elevation solitary waves were found by [11,12] for $\tau<1 / 3$. The elevation waves from the former paper are characterized by a train of non-decaying oscillations in the far field. These waves are the so-called generalized solitary waves, and it was later shown in [13] that the oscillatory tails never vanish. In this work, we examine whether the effect of electric fields can remove the far-field ripples of the generalized solitary waves.

The problem of two-dimensional free-surface capillary-gravity waves propagating on a perfectly conducting fluid under the 
effect of vertical electric fields has been investigated intensively by many authors. In the papers of [14-16] the KdV, modified KdV and KdV-Benjamin-Ono equations were derived respectively. These models were obtained in the long-wave approximation, where the depth of fluid layer is assumed to be much smaller than the typical wavelength. A comprehensive summary of the model equations can be found in [17]. Fully nonlinear travellingwave solutions were found in [18], based on a boundary integral method. It is noted that there are no studies on time-dependent solutions of the full Euler equations. However, when the fluid is assumed to be a dielectric, and the gas layer a perfect conductor, a time-dependent conformal mapping technique, first pioneered by [19], was employed in [20] to compute the dynamics of solitary waves in deep water. In this work, we generalize the results of [20] to the case of a finite-depth fluid layer and examine the destabilizing effect of the normal electric field. Both weakly nonlinear models and fully nonlinear computations are considered.

The rest of the paper is structured as follows. The problem is formulated mathematically in Section 2. The linear theory and weakly nonlinear models are derived respectively in Sections 3 and 4. The numerical scheme based on the timedependent conformal mapping is described in Section 5. The fully nonlinear results are presented and discussed in Section 6. Finally, a conclusion is given in Section 7.

\section{Formulation}

We consider the two-dimensional irrotational flow of an inviscid incompressible fluid of finite depth that is bounded above by a hydrodynamically passive region. The fluid is assumed to be a perfect dielectric with permittivity $\epsilon_{0}$. The passive region above the fluid is assumed to be perfectly conducting. This Dielectric (fluid)-Conductor (gas) system is actually a one-layer problem which can be formulated by using Cartesian coordinates with the $y$-axis directed vertically upwards, and $y=0$ at the undisturbed level. The formulation is shown in Fig. 1. The gravity $g$ and the surface tension $\sigma$ are both included in the formulation. The deformation of the free surface is denoted by $y=\zeta(x, t)$. A vertical electric field with voltage potential $v$ is applied. We assume that $v=-V_{0}$ at the bottom, where $V_{0}$ is a constant. Since the fluid motion can be described by a velocity potential function $\phi(x, y, t)$, the governing equations can then be written as

$$
\begin{array}{ll}
\nabla^{2} \phi=0, & \text { for } y<\zeta(x, t), \\
\nabla^{2} v=0, & \text { for } y<\zeta(x, t), \\
\zeta_{t}=\phi_{y}-\phi_{x} \zeta_{x}, & \text { on } y=\zeta(x, t), \\
v=0, & \text { on } y \geq \zeta(x, t), \\
v=-V_{0}, & \text { on } y=-h, \\
\phi_{y}=0, & \text { on } y=-h .
\end{array}
$$

Furthermore, it is shown in [20] that the Bernoulli equation satisfied on the free-surface gives

$$
\begin{gathered}
\phi_{t}+\frac{1}{2}|\nabla \phi|^{2}+g y-\frac{\epsilon_{0}}{\rho\left(1+\zeta_{x}^{2}\right)}\left[\frac{1}{2}\left(1-\zeta_{x}^{2}\right)\left(v_{x}^{2}-v_{y}^{2}\right)+2 \zeta_{x} v_{x} v_{y}\right] \\
-\frac{\sigma}{\rho} \frac{\zeta_{x x}}{\left(1+\zeta_{x}^{2}\right)^{3 / 2}}=0, \quad \text { on } \quad y=\zeta(x, t),
\end{gathered}
$$

where the subscripts denote partial derivatives. The last three terms of (8) are respectively the force due to gravity, the Maxwell stresses resulting from the electric field, and the force due to surface tension. Eqs. (4) and (7) are the kinematic boundary condition on the free surface and the no-penetration condition at

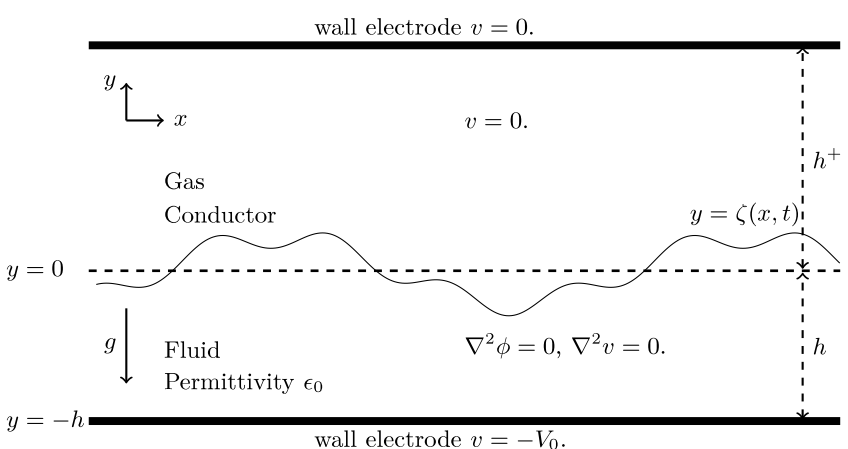

Fig. 1. Configuration of the problem. The gravity acts in the negative $y$-direction. We denote the equation of the unknown free surface by $y=\zeta(x, t)$.

the bottom. The condition (5) expresses the fact that the region above the fluid is a perfect conductor, and in turn implies

$v_{x}+v_{y} \zeta_{x}=0, \quad$ on $y=\zeta(x, t)$.

Condition (9) allows us to manipulate the electric field term in the dynamic boundary condition (8), resulting in

$$
\begin{aligned}
& \phi_{t}+\frac{1}{2}|\nabla \phi|^{2}+g \zeta+\frac{\epsilon_{0}}{2 \rho}|\nabla v|^{2}-\frac{\sigma}{\rho} \frac{\zeta_{x x}}{\left(1+\zeta_{x}^{2}\right)^{3 / 2}}=0 \quad \text { on } \\
& y=\zeta(x, t) .
\end{aligned}
$$

We choose $h, \sqrt{h / g}$ and $V_{0}$ as the reference length, time and voltage potential respectively. In this scaling, the bottom boundary is given by $y=-1$. The governing Eqs. (2) and (3) remain the same, while the dynamic boundary condition (10) becomes

$\phi_{t}+\frac{1}{2}|\nabla \phi|^{2}+\zeta+\frac{E_{b}}{2}|\nabla v|^{2}-\tau \frac{\zeta_{x x}}{\left(1+\zeta_{x}^{2}\right)^{3 / 2}}=0 \quad$ on $\quad y=\zeta(x, t)$,

where $\tau$ is the Bond number (see (1)) and $E_{b}$ is the electric Bond number defined by

$E_{b}=\frac{\epsilon_{0} V_{0}^{2}}{\rho g h^{3}}$.

The boundary conditions on the voltage potential are now scaled to be

$$
\begin{array}{ll}
v=0, & \text { on } y=\zeta(x, t), \\
v=-1, & \text { on } y=-1 .
\end{array}
$$

The kinematic conditions (4), (7) and (9) remain unchanged.

\section{Linear theory}

We linearize the system by writing

$$
\begin{aligned}
& \zeta(x, t)=A e^{i(k x-\omega t)}, \\
& \phi(x, y, t)=B e^{i(k x-\omega t)} \cosh k(y+1), \\
& v(x, y, t)=y+C e^{i(k x-\omega t)} \sinh k(y+1),
\end{aligned}
$$

where $A, B$ and $C$ are small constants. By applying condition (9), we have

$v=y-\frac{\zeta \sinh k(y+1)}{\sinh k(\zeta+1)}$.

By differentiating (11) with respect to $t$, making use of (4) and dropping all the nonlinear terms, we obtain the linear dispersion relation

$c_{p}^{2}=\left(\frac{1}{k}+\tau k\right) \tanh k-E_{b}$, 


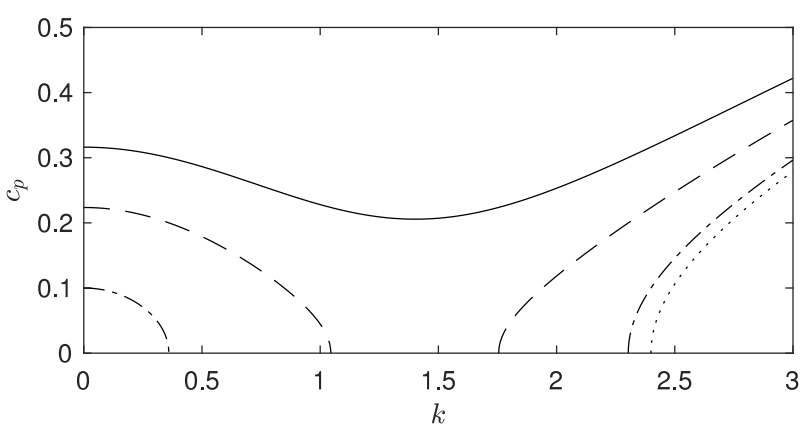

Fig. 2. Graph of the linear dispersion relation for $E_{b}=0.9$ (solid), 0.95 (dashed), 0.99 (dashed-dotted) and 1 (dotted) when $\tau=1 / 4$ and the critical value $E_{b}^{*}=0.9423$.

where $c_{p}=\omega / k$ is the phase speed. Short waves $(k \gg 1)$ are always linearly stable for a fixed $E_{b}$. However, for long waves $(k \rightarrow 0)$, we obtain $c_{p}^{2} \sim 1-E_{b}$, which indicates that long waves are linearly unstable for $E_{b}>1$. It is well known that there exists a minimum of the phase speed when $\tau<1 / 3$. In the following discussion, and throughout the paper, we choose $\tau=1 / 4$ in most of our numerical computations. This choice of $\tau$ is made such that there exists a minimum in the dispersion relation, allowing the existence of depression solitary waves and elevation generalized solitary waves, as discussed in Section 1.

When $E_{b}<E_{b}^{*} \simeq 0.9423$, the right-hand side of (19) is positive for every $k$, and there exists a minimum at $k=k^{*}=1.4026$, where the depression solitary waves bifurcate [21]. However, if $E_{b}$ becomes larger than $E_{b}^{*}$, the electric field starts to destabilize the waves with wavenumber close to $k^{*}$. When $E_{b}$ is further increased to be greater than 1 , the long waves $(k \rightarrow 0)$ are all destabilized, i.e. the $\mathrm{KdV}$ is no longer a valid model, and only short waves survive. An illustrating graph of $c_{p}$ against $k$ for different $E_{b}$ is shown in Fig. 2.

\section{Weakly nonlinear regime}

In this section, we study the weakly nonlinear regime under the long wave assumption. We derive the KdV equation by using the Dirichlet-Neumann operators for this problem, and discuss how the weakly nonlinear regime relates to the fully nonlinear computations which will be presented in Sections 5 and 6 .

\subsection{Dirichlet-neumann operators}

The Dirichlet-Neumann operator (DNO) can be defined for the fluid velocity potential as follows

$G(\zeta) \Phi=-\zeta_{x} \phi_{x}+\phi_{y}=\sqrt{1+\zeta_{x}^{2}} \frac{\partial \phi}{\partial \mathbf{n}}$,

where $\Phi(x, t) \triangleq \phi(x, \zeta, t)$ is a surface variable, and $\mathbf{n}$ is the unit normal vector pointing out of the surface. A modified voltage potential can be defined by $w=v-y$ and the kinematic condition (9) becomes

$w_{x}+\zeta_{x} w_{y}=-\zeta_{x}$.

On $y=\zeta$, we define $\mathcal{W}(x, t) \triangleq w(x, \zeta, t)=-\zeta(x, t)$. A DNO for $\mathcal{W}$ can be written as

$G^{-}(\zeta)(-\zeta)=G^{-}(\zeta) \mathcal{W}=-\zeta_{x} w_{x}+w_{y}=\sqrt{1+\zeta_{x}^{2}} \frac{\partial w}{\partial \mathbf{n}}$.

Following [22], the kinematic and the dynamic boundary condition can be rewritten in terms of the surface variables $\Phi$ and $\zeta$ by using the DNOs as follows

$\zeta_{t}=G(\zeta) \Phi$,
$\Phi_{t}=-\zeta+\tau \frac{\zeta_{x x}}{\sqrt{1+\zeta_{x}^{2}}}+M_{f}+M_{e}$

where

$M_{f}=\frac{(G(\zeta) \Phi)^{2}+2(G(\zeta) \Phi)\left(\zeta_{x} \Phi_{x}\right)-\Phi_{x}^{2}}{2\left(1+\zeta_{x}^{2}\right)}$

and

$M_{e}=-\frac{E_{b}}{2} \frac{\left(G^{-}(\zeta) \zeta\right)^{2}-2 G^{-}(\zeta) \zeta-\zeta_{x}^{2}}{1+\zeta_{x}^{2}}$.

It was shown by [23] that the DNOs are analytic provided the $L^{\infty}$. norm and Lipschitz-norm of the displacement $\zeta$ are smaller than a certain constant. They can be expanded in the Taylor series

$G(\zeta)=\sum_{n=0}^{\infty} G_{n}(\zeta)$

$G^{-}(\zeta)=\sum_{n=0}^{\infty} G_{n}^{-}(\zeta)$

where $G_{n}$ and $G_{n}^{-}$are homogeneous of order $n$ in $\zeta$. Applying $G$ to the ground state solution $e^{i k x} \cosh k(y+1)$ of the fluid velocity potential and $G^{-}$to $e^{i k x} \sinh k(y+1)$ of the voltage potential, the first two terms of the series can be obtained after performing similar calculations as to those in $[17,22]$

$G_{0}=\mathcal{D} \tanh (\mathcal{D})$,

$G_{0}^{-}=\mathcal{D} \operatorname{coth}(\mathcal{D})$,

$G_{1}(\zeta)=\mathcal{D} \zeta \mathcal{D}-G_{0} \zeta G_{0}$

$G_{1}^{-}(\zeta)=\mathcal{D} \zeta \mathcal{D}-G_{0}^{-} \zeta G_{0}^{-}$,

where $\mathcal{D}=-i \partial_{x}$. By ignoring the terms of $o\left(\zeta^{2}\right)$, the governing system is reduced to

$\zeta_{t}=G_{0} \Phi+G_{1}(\zeta) \Phi$

$\Phi_{t}=-\zeta+\tau \frac{\zeta_{x x}}{\sqrt{1+\zeta_{x}^{2}}}+\frac{1}{2}\left[\left(G_{0} \Phi\right)^{2}-\Phi_{x}^{2}\right]$
$-\frac{E_{b}}{2}\left[\left(G_{0}^{-} \zeta\right)^{2}-2 G_{0}^{-} \zeta-2 G_{1}^{-}(\zeta) \zeta-\zeta_{x}^{2}\right]$.

\subsection{Long-wave models}

In this subsection, we derive the model equation under the long wave limit, i.e. assuming that the typical wavelength is much greater than the depth of the fluid layer. The displacement $\zeta$ and the velocity potential function are assumed to be small. Their size is measured by a small parameter $\epsilon$. We consider the following scaling:

$\zeta=O\left(\epsilon^{2}\right), \quad \Phi=O(\epsilon), \quad \partial_{x}=O(\epsilon)$,

$\partial_{t}=O(\epsilon), \quad \tau=O(1), \quad E_{b}=O(1)$.

Eqs. (29) and (30) can be expanded as polynomials in $\mathcal{D}$

$G_{0}=\mathcal{D}^{2}-\frac{1}{3} \mathcal{D}^{4}+\frac{2}{15} \mathcal{D}^{6}+O\left(\epsilon^{8}\right)$,

$G_{1}=\mathcal{D} \zeta \mathcal{D}-\mathcal{D}^{2} \zeta \mathcal{D}^{2}+O\left(\epsilon^{8}\right)$,

$G_{0}^{-}=I_{d}+\frac{1}{3} \mathcal{D}^{2}-\frac{1}{45} \mathcal{D}^{4}+O\left(\epsilon^{6}\right)$,

$G_{1}^{-}=-\zeta+\mathcal{D} \zeta \mathcal{D}-\frac{1}{3} \mathcal{D}^{2} \zeta-\frac{1}{3} \zeta \mathcal{D}^{2}+\zeta^{2}+O\left(\epsilon^{6}\right)$.

The governing system reads

$\zeta_{t}=-\Phi_{x x}-\frac{1}{3} \Phi_{x x x x}-\zeta \Phi_{x x}-\zeta_{x} \Phi_{x}$ 
$\Phi_{t}=-\frac{1}{2} \Phi_{x}^{2}-\left(1-E_{b}\right) \zeta+\left(\tau-\frac{E_{b}}{3}\right) \zeta_{x x}-\frac{3 E_{b}}{2} \zeta^{2}$

which is a Boussinesq-type system in $\zeta$ and $\Phi$. A one-dimensional Benney-Luke type equation can be obtained by differentiating (40) with respect to $t$ and combining this with (39) to find

$\Phi_{t t}-c_{0}^{2} \Phi_{x x}+\left(\tau-\frac{1}{3}\right) \Phi_{x x x x}+\left(\Phi_{x}^{2}\right)_{t}+\left(1+\frac{3 E_{b}}{c_{0}^{2}}\right) \Phi_{t} \Phi_{x x}=0$,

where $c_{0}^{2} \zeta=-\Phi_{t}+O\left(\epsilon^{4}\right)$ has been used. Here, $c_{0}=\sqrt{1-E_{b}}$ is the long wave speed which can be obtained by taking the limit $k \rightarrow 0$ in the linear dispersion relation (19). To get the uni-directional Korteweg de Vries equation (KdV), we introduce

$X=\epsilon\left(x-c_{0} t\right), \quad T=\epsilon^{3} t$.

Changing the variables from $(x, t)$ to $(X, T)$ in (41) and keeping only the terms up to $O\left(\epsilon^{5}\right)$ yields

$\Phi_{X T}-\frac{1}{2 c_{0}}\left(\tau-\frac{1}{3}\right) \Phi_{X X X X}+\frac{3}{2}\left(1+\frac{E_{b}}{c_{0}^{2}}\right) \Phi_{X} \Phi_{X X}=0$.

Transforming back to the original variables, the celebrated $\mathrm{KdV}$ is obtained

$q_{t}+c_{0} q_{x}-\frac{1}{2 c_{0}}\left(\tau-\frac{1}{3}\right) q_{x x x}+\frac{3}{2}\left(1+\frac{E_{b}}{c_{0}^{2}}\right) q q_{x}=0$,

where $q=\Phi_{x}$. When $\tau=1 / 3$, it is not difficult to obtain a fifth-order KdV equation as follows

$q_{t}+c_{0} q_{x}+\frac{1}{90 c_{0}} q_{x x x x x}+\frac{3}{2}\left(1+\frac{E_{b}}{c_{0}^{2}}\right) q q_{x}=0$.

The derivation can be easily extended for three-dimensional waves, where a Kadomtsev-Petviashvili equation will be obtained. The readers are referred to [17] for more details.

\subsection{Connection to other EHD problems}

Many other electrohydrodynamic problems of free-surfaces have been investigated in the last decade. In particular, the problem of Perfect Conductor (fluid)-Dielectric (gas) received much attention [10,16-18,24]. The associated linear dispersion relation under our scaling is

$c_{p}^{2}=\left(\frac{1}{k}+\tau k-E_{b} \operatorname{coth} R k\right) \tanh k$,

where $R=h^{+} / h$ is the ratio of the depths of the two layers. The corresponding $\mathrm{KdV}$ equation for $R \gg 1$ is

$q_{t}+q_{x}-\frac{E_{b}}{2} Q q_{x}+\frac{1}{2}\left(\frac{1}{3}-\tau\right) q_{x x x}+\frac{3}{2} q q_{x}=0$,

where $Q$ is a pseudo-differential operator, defined by

$Q=\sqrt{-\partial_{x x}} \operatorname{coth}\left(R \sqrt{-\partial_{x x}}\right)$.

We note that $Q$ reduces to $\mathcal{H} \partial_{x}$ when $R \rightarrow \infty$, i.e. the upper region is infinitely deep, with $\mathcal{H}$ being the Hilbert transform defined by

$\mathcal{H}[f](\xi)=\frac{1}{\pi} \int \frac{f\left(\xi^{\prime}\right)}{\xi^{\prime}-\xi} d \xi^{\prime}$.

Then (47) reduces to a KdV-Benjamin-Ono equation [16]

$q_{t}+q_{x}-\frac{E_{b}}{2} \mathcal{H}\left[q_{x x}\right]+\frac{1}{2}\left(\frac{1}{3}-\tau\right) q_{x x x}+\frac{3}{2} q q_{x}=0$.

We note that (50) can be generalized to a Benjamin-Ono Kadomtsev-Petviashvili equation in three-dimensional problems (see $[17,25]$ ).
It is of interest to note that, in the particular case $R=1$, i.e. the upper and the lower region are of the same size, the linear dispersion relation (46) is exactly the same as (19) for the case of Dielectric-Perfect Conductor. Under the long-wave limit, $Q$ can be expanded as a Taylor series in $D$ as shown in Section 4.2. Eq. (47) reduces to

$q_{t}+c_{0} q_{x}-\frac{1}{2 c_{0}}\left(\tau-\frac{1}{3}\right) q_{x x x}+\frac{3}{2}\left(1-\frac{E_{b}}{c_{0}^{2}}\right) q q_{x}=0$.

The linear terms match with those from (44), as expected.

\section{Numerical scheme}

To solve the fully nonlinear equations numerically, we employ the time-dependent conformal mapping technique. It is a method pioneered by [19], which maps the free surface onto the horizontal axis in a new complex plane denoted by $(\xi, \eta)$. The fluid domain is transformed onto a strip with depth $D$. The harmonic conjugate of $x(\xi, \eta)$ can be obtained via the CauchyRiemann equations for the analytic function $z(\xi, \eta)=x(\xi, \eta)+$ $i y(\xi, \eta)$. Similarly, we can derive the harmonic conjugates of $\phi(\xi, \eta)$ and $v(\xi, \eta)$, denoted by $\psi(\xi, \eta)$ and $v(\xi, \eta)$ respectively. In the transformed plane, we write the surface variables as $X(\xi, t) \equiv$ $x(\xi, 0, t), Y(\xi, t) \equiv y(\xi, 0, t), \Phi(\xi, t) \equiv \phi(\xi, 0, t), \Psi(\xi, t) \equiv$ $\psi(\xi, 0, t), V(\xi, t) \equiv v(\xi, 0, t)$ and $N(\xi, t) \equiv v(\xi, 0, t)$. The map can be formally defined as the solution of the following boundary value problem

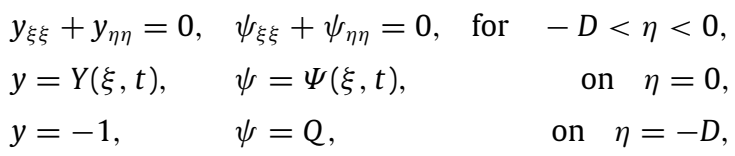

where $Y(\xi, t)=\zeta(\xi, 0, t) \cdot Q$ is an arbitrary constant, and we choose $Q=\langle\Psi\rangle$, where $\langle\cdot\rangle$ is the mean value defined as

$\langle f\rangle=\frac{1}{L} \int_{-L / 2}^{L / 2} f(\xi) d \xi$,

where $\left[-\frac{L}{2}, \frac{L}{2}\right]$ is the computational domain, and $L$ is usually chosen to be the wavelength. It can be shown that

$D=1+\langle Y\rangle$,

$X_{\xi}=1-\mathcal{T}\left[Y_{\xi}\right]$,

$\Psi_{\xi}=\mathcal{T}\left[\Phi_{\xi}\right]$,

$N_{\xi}=-\frac{1}{D}+\mathcal{T}\left[V_{\xi}\right]$,

where $\mathcal{T}[$.] is defined by

$\mathcal{T}[f](\xi)=\frac{1}{2 D} \mathrm{PV} \int f\left(\xi^{\prime}\right) \operatorname{coth}\left(\frac{\pi}{2 D}\left(\xi^{\prime}-\xi\right)\right) d \xi^{\prime}$.

Here 'PV' denotes the Cauchy principal value of the integral. We note that $V_{\xi}=0$ as $v$ is identically zero everywhere on the free surface. Next, we follow [26] to derive the time-evolution equations, which finally read

$$
\begin{aligned}
Y_{t}= & Y_{\xi} \mathcal{T}\left[\frac{\Psi_{\xi}}{J}\right]-X_{\xi} \frac{\Psi_{\xi}}{J}, \\
\Phi_{t}= & \frac{1}{2 J}\left(\Psi_{\xi}^{2}-\Phi_{\xi}^{2}\right)-Y-\frac{E_{b}}{2 D^{2} J}+\tau \frac{X_{\xi} Y_{\xi \xi}-Y_{\xi} X_{\xi \xi}}{J^{3 / 2}} \\
& +\Phi_{\xi} \mathcal{T}\left[\frac{\Psi_{\xi}}{J}\right],
\end{aligned}
$$

where $J=X_{\xi}^{2}+Y_{\xi}^{2}$ is the Jacobian of the conformal map. 

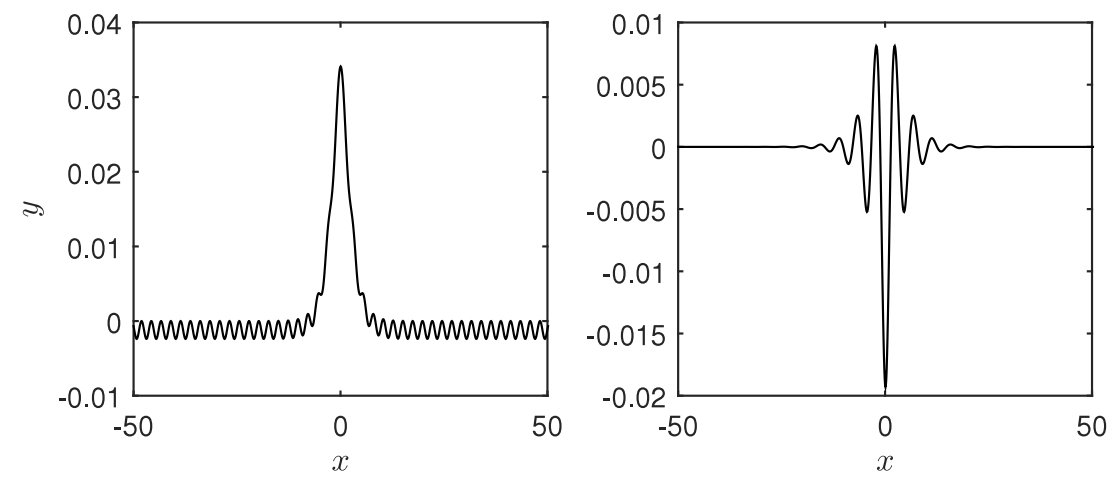

Fig. 3. Solitary wave profiles for $\tau=1 / 4$ in the absence of electric fields.

For travelling waves, all functions depend on $x-c t$, where $c$ is an unknown constant. After similar calculations as those presented in [27], we have

$\Psi=c Y$.

Then the resulting governing equation becomes

$\frac{1}{2}\left(c^{2}+\frac{E_{b}}{D^{2}}\right)\left(\frac{1}{J}-1\right)+Y-\tau \frac{X_{\xi} Y_{\xi \xi}-Y_{\xi} X_{\xi \xi}}{J^{3 / 2}}=0$.

In the present paper, solitary waves are approximated by long periodic waves. It follows that $D$ is needs to be updated over time in unsteady simulations to ensure that the wavelength in the conformal space is the same as that in the physical space. The surface elevation can be expressed as a Fourier series

$Y(\xi)=\sum_{n=1}^{N} a_{n} \cos \left(\frac{2 n \pi \xi}{L}\right)+b_{n} \sin \left(\frac{2 n \pi \xi}{L}\right)$,

where the coefficients $a_{n}, b_{n}$ are unknowns. By imposing symmetry at $X=0$, all the sin terms vanish, i.e. $b_{n}$ are zero for arbitrary $n$. The $\mathcal{T}$-transform is computed numerically by Fourier multipliers as follows

$\mathcal{T}[g]=\mathcal{F}^{-1}[i \operatorname{coth}(k D) \mathcal{F}[g]]$,

where $\mathcal{F}$ is the Fourier transform. In most computations, we use 2048 Fourier modes and $L=100$ to achieve a high computing accuracy. This numerical scheme has been successfully used in the context of gravity waves [28] and flexural-gravity waves [29] on water of finite depth.

\section{Numerical results}

\subsection{Travelling waves}

The fully nonlinear problem is solved by using the numerical scheme introduced in Section 5 . We start by computing solitary waves for $\tau=1 / 4$ in the absence of electric fields, i.e. capillary-gravity waves. We manage to reproduce the results of [11] for depression solitary waves and elevation generalized solitary waves. Two typical wave profiles are depicted in Fig. 3. Next, we include a normal electric field with strength measured by $E_{b}$. To examine the long wave model obtained in Section 4.2, we compute depression solitary waves with speed close to $c_{0}$ for $E_{b}=0.5$ and $\tau=1 / 3$ by using the full Euler and the fifth-order KdV equation. The results matched quite well as shown in Fig. 4.

Next, we restrict our attention to the solitary waves for $\tau=$ $1 / 4$ in the presence of a normal electric field, and study numerically the bifurcations as well as their dynamics. The complete amplitude-speed diagram for solitary waves is presented in Fig. 5 for $E_{b}=0.5$, and some corresponding profiles are shown in

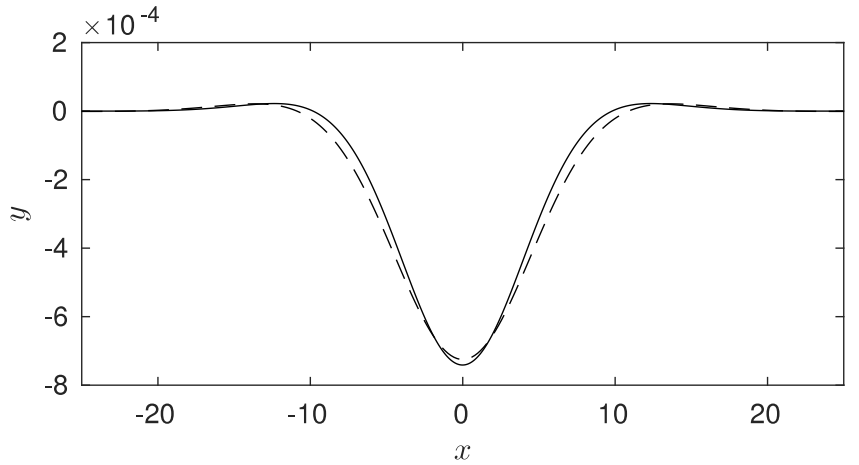

Fig. 4. Comparison between the numerical solutions for a depression wave with $c=0.7068$ for $\tau=1 / 3$ and $E_{b}=0.5$ by the full Euler equation (solid) and the fifth-order $\mathrm{KdV}$ equation (dashed).

Fig. 6. The branch of depression waves starts at the minimum of the dispersion curve $\left(c=c_{\min }\right)$, and decreases monotonically to $c=0$. When there is no electric field, [11] showed that the static (i.e. no flow) solitary capillary-gravity wave obtained was self-intersecting. It is found that the electric field has the effect of suppressing overhanging, as sketched in Fig. 6a. When the strength of the electric field is increased, the amplitude of the static depression wave decreases and shrinks to zero at the critical value of $E_{b}^{*}$, as shown in Fig. 7. For $E_{b}>E_{b}^{*}$, the regime becomes linearly unstable and therefore no solitary waves exist for such values of $E_{b}$. The curve of depression waves from Fig. 5 emanates from zero amplitude at $c=c_{\min }$, which suggests that the associated nonlinear Schrödinger equation is focussing, and therefore wavepacket solitary waves bifurcate from infinitesimal periodic waves. The one-dimensional stability can be examined by imposing an initial longitudinal perturbation to the solitary wave. An example is presented in Fig. 8, where the wave is perturbed by $-5 \%$ in amplitude at $t=0$ and placed in a frame of reference moving with the initial phase speed. As time increases, the wave travels towards the left of the frame since the depression wave of smaller amplitude propagates faster, as can be seen from the amplitude-speed diagram 5 . Such numerical tests were performed to all the solutions from the depression branch, and no one-dimensional instabilities were observed. For elevation waves, the so-called generalized solitary waves with non-decaying oscillatory ripples at the tail are found. These waves also bifurcate from infinitesimal periodic waves, but at a speed $c_{0}^{\prime}\left(>c_{\text {min }}\right)$. The value of $c_{0}^{\prime}$ depends on the value of $L$ but if the periodicity is suspended, i.e. $L \rightarrow \infty$ and $k \rightarrow 0, c_{0}^{\prime}$ tends to $c_{0}=\sqrt{1-E_{b}}$ which equals 0.7071 for $E_{b}=0.5$. As the dispersion relation admits a minimum, these waves appear due to the resonance of the long wave mode $k=0$ with periodic 

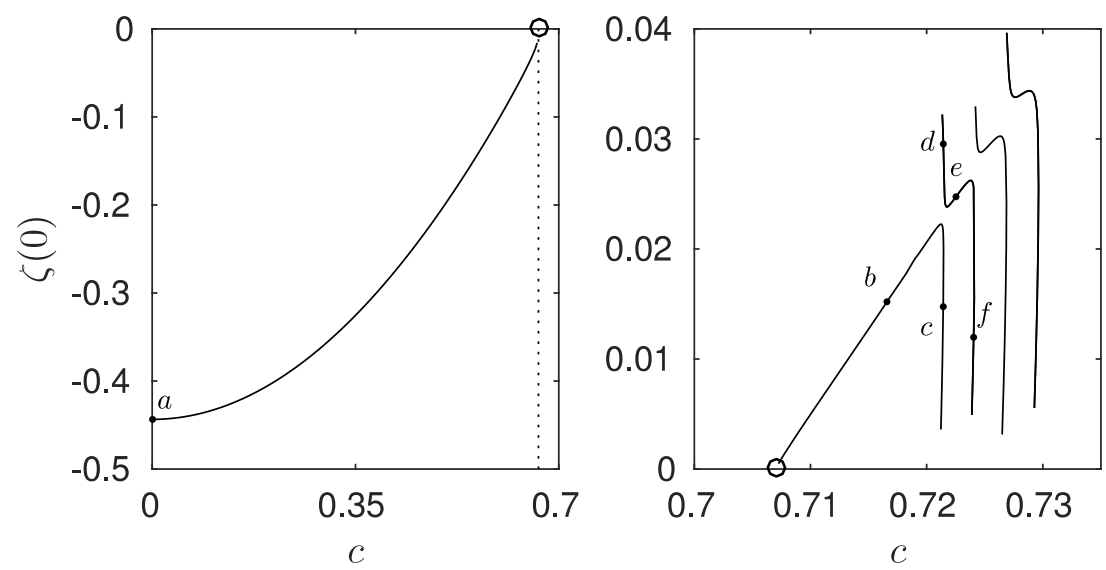

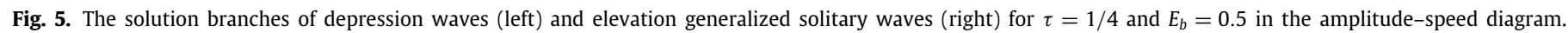
The bifurcation points are marked as circles.
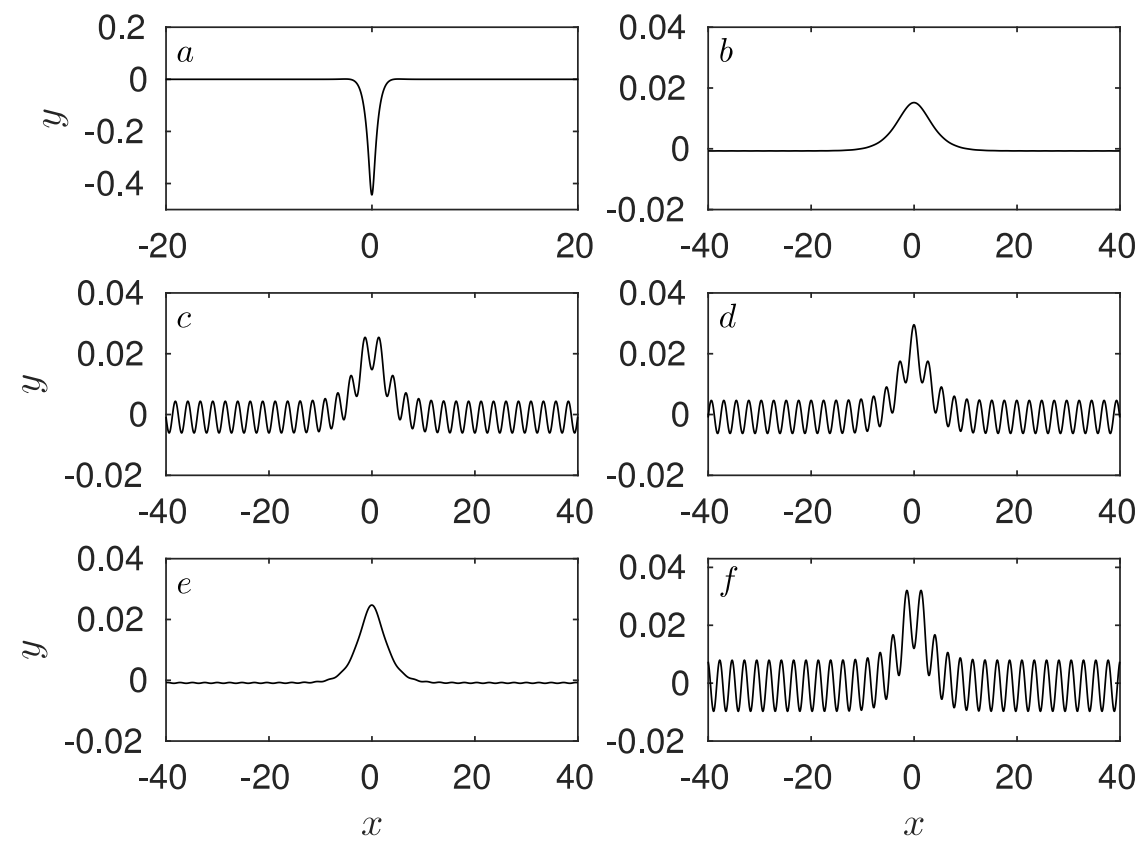

Fig. 6. The wave profiles that correspond to the points indicated in Fig. 5.

waves with wavenumber $k^{\dagger}$ propagating at the long wave speed, i.e. $c_{p}\left(k^{\dagger}\right)=c_{0}$. Although solution $b$ looks like a KdV soliton, it still has a very small non-decaying tail. Due to the assumption of periodicity, there exists infinitely many generalized solitarywave branches for a fixed computational domain. Jumping to the next branch on the right adds an additional far-field oscillation to the wave in a half wavelength (see e.g. Fig. 6 c\&f). To carry out a more rigorous investigation on whether true elevation solitary waves exist in the presence of a normal electric field, we follow $[13,30]$ to perform a numerical investigation by monitoring the curvature of the solution at the right end of the computational domain, denoted by $\kappa_{0}$, for a fixed $\tau$ and various $E_{b}$. As can be seen clearly from Fig. 9, the solution branches are all u- or n-shaped and move away from zero as we increase $E_{b}$. Therefore the value of $\kappa_{0}$ never goes to zero, i.e. generalized solitary waves do not approach KdV-like elevation soliton as a limit in the presence of electric fields. This is a complement to the work by [13] demonstrating a numerical evidence that capillarygravity elevation solitary waves do not exist on water of finite depth either in the presence or absence of electric stress. The one-dimensional stability can be studied in the same manner as the depression case, provided the value of $L$ is sufficiently large. An initial amplitude-decreasing perturbation by $5 \%$ is imposed. True generalized solitary waves (which have infinite energy) are expected to be unstable. However we have not observed any onedimensional instabilities from the numerical results for time $t$ up to 4000 as shown in Fig. 10. This is a numerical issue that may be due to the periodic assumption made in the numerical scheme which restricts the energy to be finite.

\subsection{Touch-down singularity}

In this subsection we examine numerically the linear instability which occurs when $E_{b}>E_{b}^{*}$ by using the method introduced in Section 5. A touch-down singularity is expected, due to the finite depth of the fluid, and increase in amplitude of the interface. A stable capillary-gravity depression wave (see the top left graph of Fig. 11) is chosen as the initial state with the strength of $E_{b}$ varying in time as follows

$E_{b}=0.1(j-1), \quad$ for $t \in[5(j-1), 5 j), \quad j=1,2,3, \ldots, 11$,

where $t$ is the time variable. A moving frame of reference is chosen such that the solitary wave is steady for $E_{b}=0$. When 


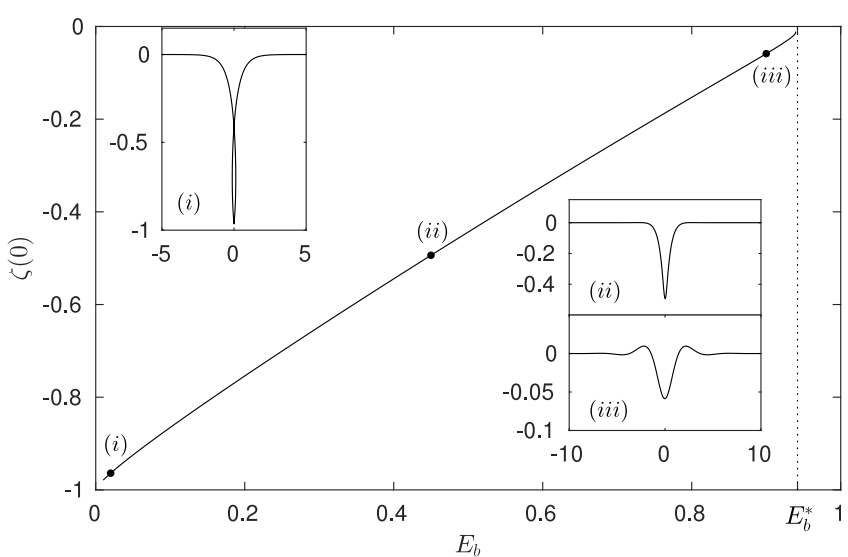

Fig. 7. Graph of $\zeta(0)$ against $E_{b}$ for depression solitary waves with zero propagating speed.

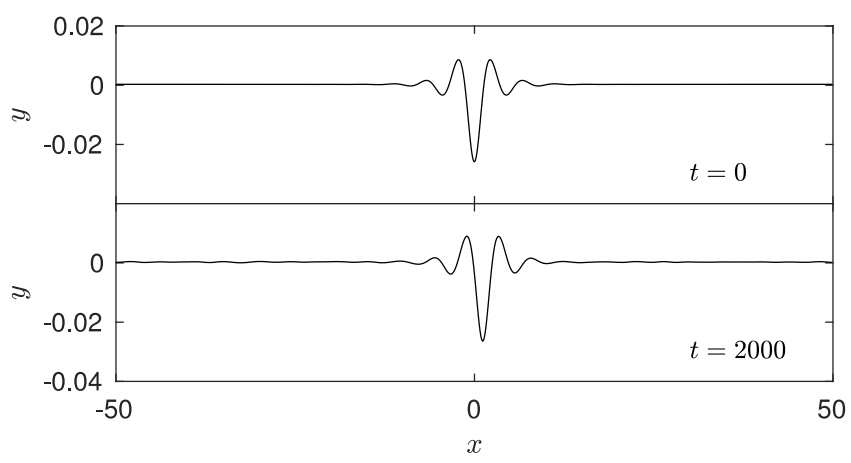

Fig. 8. Dynamics of a stable depression wave with $c=0.6566, \tau=1 / 4$, $E_{b}=0.5$. An amplitude-decreasing perturbation is initially applied. A reference frame moving with $c=0.6566$ is chosen.

the electric field is switched on and $E_{b}<E_{b}^{*}$, the solitary wave remains stable. From the left column of Fig. 11, it is observed that there is energy radiating in the form of small ripples because of the discrete jumps in $E_{b}$. There will be less radiation if the electric field is changed more gradually. Meanwhile, the speed of the solitary wave is slower, and the wave amplitude becomes larger as the electric field strength is increased, which agrees

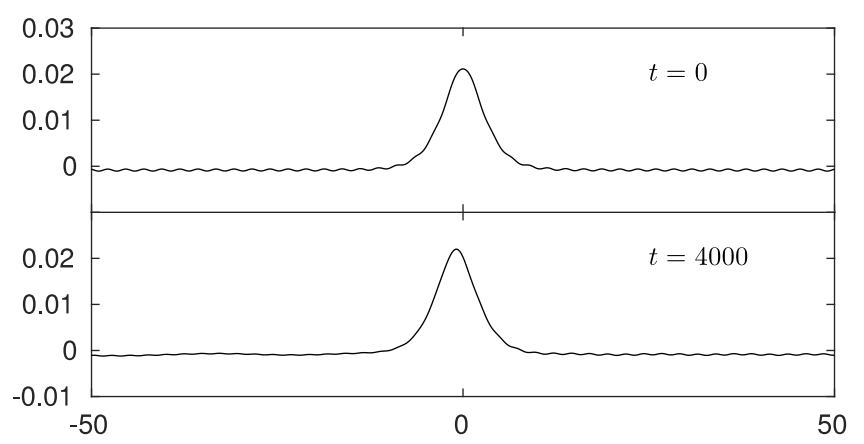

Fig. 10. Dynamics of an elevation generalized solitary wave with $c=0.7212$ $\tau=1 / 4, E_{b}=0.5$. An amplitude-decreasing perturbation is initially applied. A reference frame moving with $c=0.7212$ is chosen. Only part of the domain is shown for a better display.

with the theory. When $E_{b}>E_{b}^{*}(t \geq 50)$, it can be seen from the graphs on the right of Fig. 11 that the wave amplitude increases very quickly, and ultimately approaches the bottom. At $t>51.97$, a numerical instability is observed due to an infinite curvature where the surface collapses with the lower boundary. We call this phenomenon a touch-down singularity, where the wave is destabilized by the electric field. Such numerical experiment of destabilization can be applied to other waves such as periodic waves or generalized solitary waves. Similar touch-down singularities are expected.

\subsection{Excitation}

In Section 6.1, the one-dimensional stabilities were examined. To excite the stable solitary waves, we perform a numerical experiment by adding an external moving Gaussian pressure distribution into the Bernoulli equation, which is defined as follows

$\mathcal{P}=0.03 e^{-(x+200-U t)^{2}}$,

with $E_{b}=0.5$ fixed and $U=0.64$, which is chosen to be close to the phase speed minimum. The pressure is initially switched on at $x=-200$ and later removed at $t=20$. We let the numerical experiment continue for a long time (up to $t=400$ ). A stable depression wave propagating with speed $0.5836\left(<c_{\min }\right)$
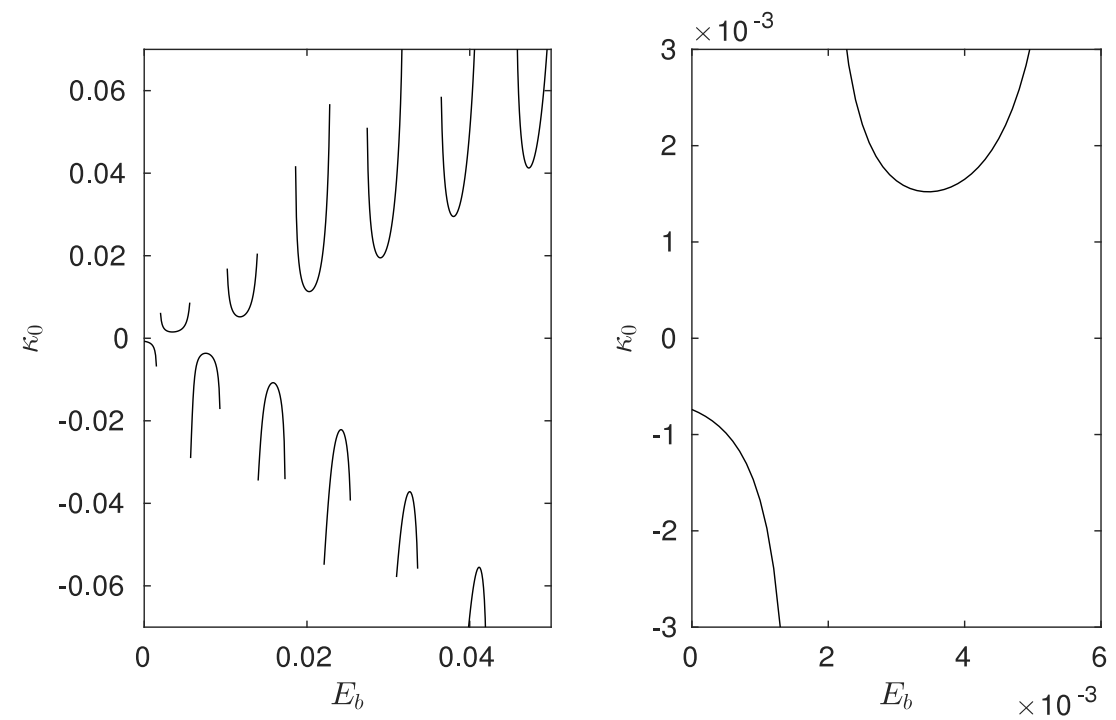

Fig. 9. (Left) Value of $\kappa_{0}$ versus the electric field strength $E_{b}$ when $\tau=1 / 4$. (Right) A blow-up graph near $E_{b}=0$. 

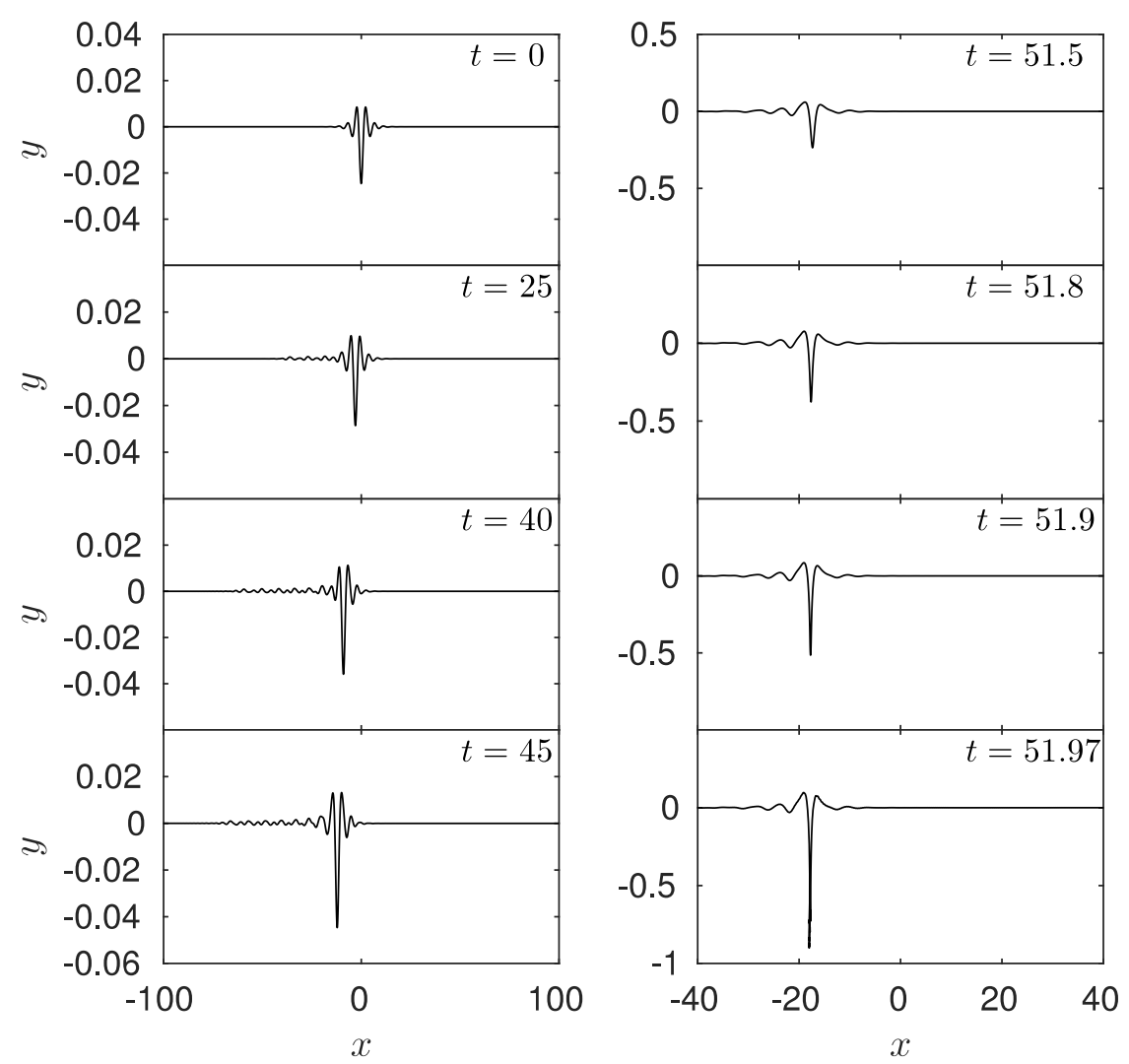

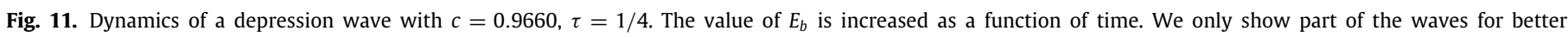
display.

is obtained. As the surface is depressed below the mean level locally by an external pressure, due to the conservation of mass in the computational domain, it must be elevated above the mean level somewhere. We have observed that such elevation propagates in the form of a generalized solitary wave travelling with speed 0.7347 ( $>c_{0}$ ) which is faster than the speed of the depression wave. Hence it appears in the front and gets away from the depression wave in time. The propagating wave speeds are measured from the numerical experiment by computing the mean velocities over a time interval $t \in[200,400]$. The two waves are highlighted in the bottom snapshot of Fig. 12. They are compared to the travelling solitary wave solutions computed in Section 6.1, and a strong agreement can be seen, as shown in Fig. 13.

In the above numerical experiment, after the excitation of the solitary waves, one can decrease progressively $E_{b}$ to 0 , such that the solitary waves are classic capillary-gravity waves. These waves can also be excited without the use of an electric field. We repeat the experiment, this time taking $E_{b}=0$ with a disturbance $P$ defined by (68) with $U=0.96$. The pressure is switched off at $t=20$, and we see again the formation of a depression wave and an elevation generalized solitary wave (see Fig. 14). However, it can be observed from the two experiments of excitation for $E_{b}=0$ and $E_{b}=0.5$ that the speed difference between the solitary waves becomes greater in the presence of the electric field, which in turn makes the two waves further apart from each other in the same period of time. In fact, the generated depression wave travels with a speed less than and close to $c_{\min }$, while the excited generalized solitary wave travels with a speed faster than and close to $c_{0}$. For a reasonable value of $E_{b}$, the difference $c_{0}-c_{\min }$ becomes greater than the non-electric case, which indicates that the electric field is useful for separating the excited solitary waves in a short time.

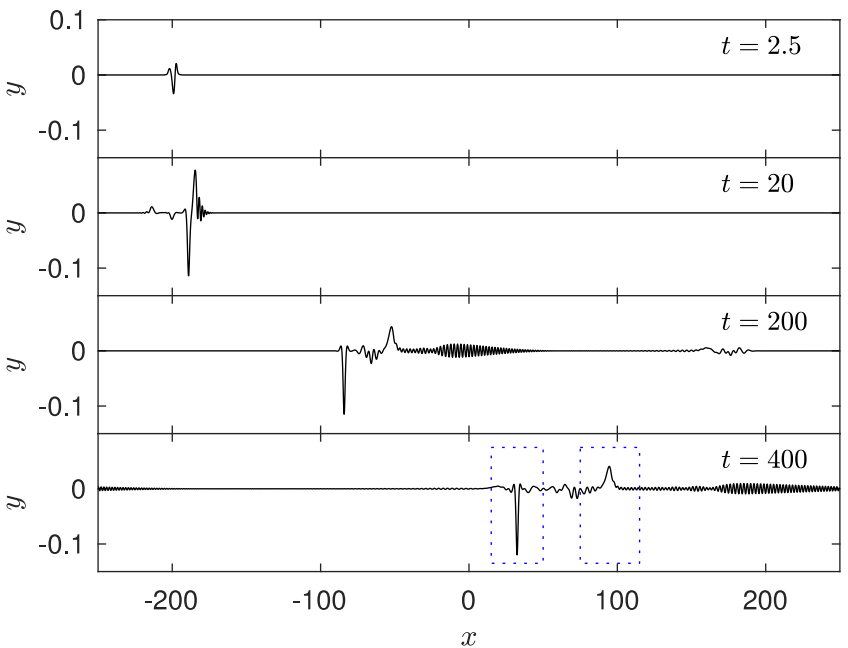

Fig. 12. Snapshots of the excitation experiment with $E_{b}=0.5$ at time $t=2.5$, 20,200 and 400. A disturbance moving with speed 0.64 is switched on at $t=0$ and off at $t=20$. At the end of the experiment, a depression wave moving with speed 0.5836 and an elevation generalized solitary wave with speed 0.7347 are obtained.

\section{Conclusion}

In the current work, the problem of electrohydrodynamic capillary-gravity waves on a dielectric fluid under a normal electric field was investigated. Linear and weakly nonlinear theory were both presented. Long-wave model equations were derived by using the Dirichlet-Neumann operators. Fully nonlinear computations were carried out for solitary waves and their dynamics. 

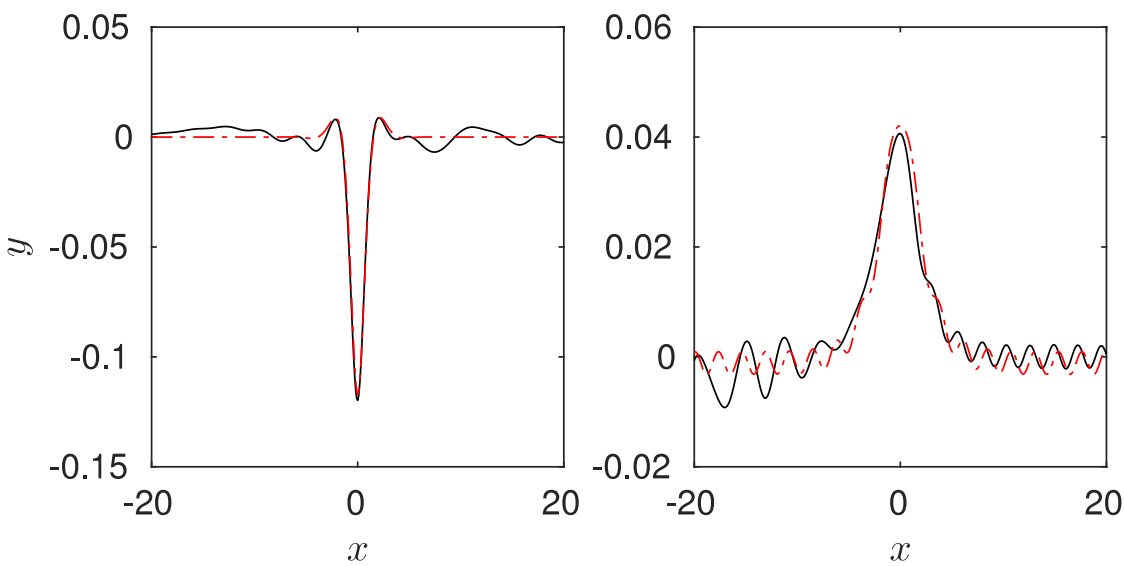

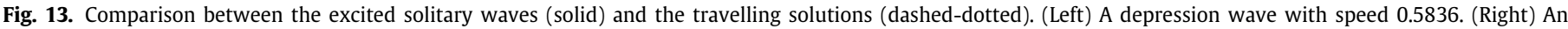
elevation generalized solitary wave with speed 0.7347 . We only show part of the waves for better display.

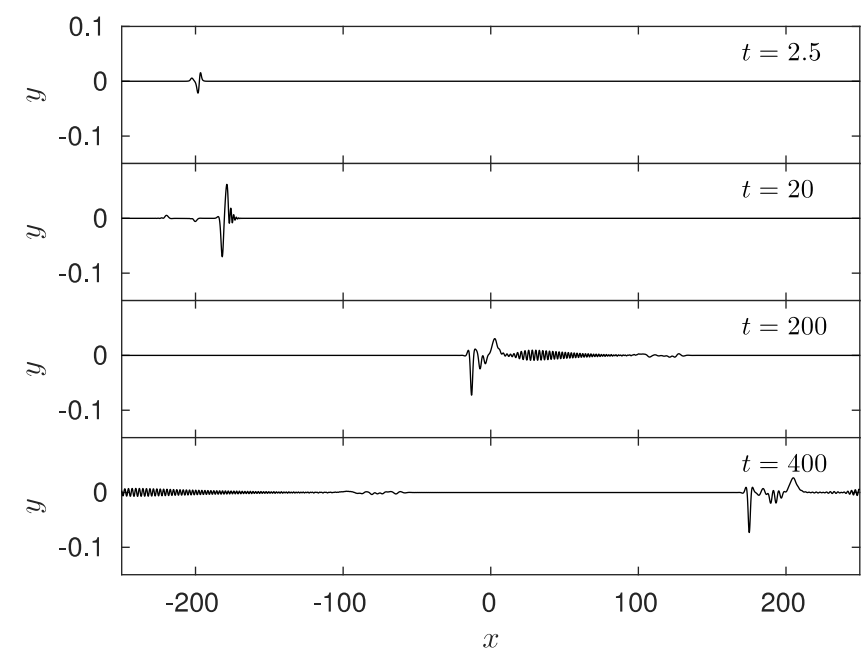

Fig. 14. Snapshots of the excitation experiment with $E_{b}=0$ at time $t=2.5$, 20,200 and 400. A disturbance moving with speed 0.96 is switched on at $t=0$ and off at $t=20$. At the end of the experiment, a depression wave moving with speed 0.9385 and an elevation generalized solitary wave with speed 1.0391 are obtained.

A numerical experiment of excitation was conducted to generate the solitary waves. The comparisons between the excited and steady solutions were drawn, and an excellent agreement was obtained.

\section{Acknowledgements}

The author T.G. would like to thank the EPSRC UK Fluids Network and Department of Mathematics, UCL for supporting a Short Research Visit which made this work possible. A.D. was supported by EPSRC, UK under grant EP/M507970/1. J.-M.V.-B. was supported in part by EPSRC, UK under grant EP/N018559/1. Z.W. was supported by the National Natural Science Foundation of China (No.11772341), the Key Research Program of Frontier Sciences, CAS, China (No. QYZDBSSW-SYS015), and the Strategic Priority Research Program of the Chinese Academy of Sciences, China (No. XDB22040203). Z.W. would also like to acknowledge the support from CAS Center for Excellence in Complex System Mechanics.

\section{References}

[1] E.M. Griffing, S.G. Bankoff, M.J. Miksis, R.A. Schluter, Electrohydrodynamics of thin flowing films, J. Fluids Eng. 128 (2) (2006) 276-283.
[2] S.F. Kistler, P.M. Schweizer, Liquid Film Coating - Scientific Principles and their Technological Implications, Chapman and Hall, 1997.

[3] G. Taylor, A. McEwan, The stability of a horizontal fluid interface in a vertical electric field, J. Fluid Mech. 22 (1) (1965) 1-15.

[4] J.R. Melcher, W.J. Schwarz, Interfacial relaxation overstability in a tangential electric field, Phys. Fluids 11 (12) (1968) 2604-2616.

[5] L.L. Barannyk, D.T. Papageorgiou, P.G. Petropoulos, Suppression of Rayleigh-Taylor instability using electric fields, Math. Comput. Simulation 82 (6) (2012) 1008-1016.

[6] R. Cimpeanu, D.T. Papageorgiou, P.G. Petropoulos, On the control and suppression of the rayleigh-taylor instability using electric fields, Phys. Fluids 26 (2) (2014) 022105

[7] A.R.F. Elhefnawy, Nonlinear electrohydrodynamic kelvin-helmholtz instability under the influence of an oblique electric field, Physica A 182 (3) (1992) 419-435.

[8] N.M. Zubarev, E. Kochurin, Nonlinear dynamics of the interface between fluids at the suppression of kelvin-helmholtz instability by a tangential electric field, JETP Lett. 104 (4) (2016) 275-280.

[9] D.T. Papageorgiou, J.-M. Vanden-Broeck, Large-amplitude capillary waves in electrified fluid sheets, J. Fluid Mech. 508 (2004) 71-88.

[10] D.T. Papageorgiou, P.G. Petropoulos, J.-M. Vanden-Broeck, Gravity capillary waves in fluid layers under normal electric fields, Phys. Rev. E 72 (5) (2005) 051601.

[11] J. Hunter, J.-M. Vanden-Broeck, Solitary and periodic gravity-capillary waves of finite amplitude, J. Fluid Mech. 134 (1983) 205-219.

[12] F. Dias, D. Menasce, J.-M. Vanden-Broeck, Numerical study of capillarygravity solitary waves, Eur. J. Mech. B Fluids 15 (1996) 17-36.

[13] A. Champneys, J.-M. Vanden-Broeck, G. Lord, Do true elevation gravitycapillary solitary waves exist? a numerical investigation, J. Fluid Mech 454 (2002) 403-417.

[14] C. Easwaran, Solitary waves on a conducting fluid layer, Phys. Fluids 31 (11) (1988) 3442-3443.

[15] T. Perelman, A. Fridman, M. Eliashevich, Modified korteweg-de vries equation in electrohydrodynamics, Zh. Eksperimental Teoreticheskoi Fiziki 66 (1974) 1316-1323.

[16] H. Gleeson, P. Hammerton, D. Papageorgiou, J.-M. Vanden-Broeck, A new application of the Korteweg-de Vries Benjamin-Ono equation in interfacial electrohydrodynamics, Phys. Fluids 19 (3) (2007) 031703.

[17] Z. Wang, Modelling nonlinear electrohydrodynamic surface waves over three-dimensional conducting fluids, Proc. R. Soc. Lond. Ser. A Math. Phys. Eng. Sci. 473 (2200) (2017) 201608.

[18] D.T. Papageorgiou, J.-M. Vanden-Broeck, Numerical and analytical studies of non-linear gravity-capillary waves in fluid layers under normal electric fields, IMA J. Appl. Math. 72 (6) (2006) 832-853.

[19] A.I. Dyachenko, E.A. Kuznetsov, M. Spector, V.E. Zakharov, Analytical description of the free surface dynamics of an ideal fluid (canonical formalism and conformal mapping), Phys. Lett. A 221 (1-2) (1996) 73-79.

[20] T. Gao, P. Milewski, D. Papageorgiou, J.-M. Vanden-Broeck, Dynamics of fully nonlinear capillary-gravity solitary waves under normal electric fields, J. Eng. Math. 108 (1) (2018) 107-122.

[21] A. Shabat, V. Zakharov, Exact theory of two-dimensional self-focusing and one-dimensional self-modulation of waves in nonlinear media, Soviet Phys. JETP 34 (1) (1972) 62.

[22] W. Craig, C. Sulem, Numerical simulation of gravity waves, J. Comput. Phys 108 (1) (1993) 73-83.

[23] R. Coifman, Y. Meyer, Nonlinear harmonic analysis and analytic dependence, pseudodifferential operators and applications 43 (12) (1985) 71-78. 
[24] P. Hammerton, Existence of solitary travelling waves in interfacial electrohydrodynamics, Wave Motion 50 (4) (2013) 676-686.

[25] M. Hunt, J.-M. Vanden-Broeck, D. Papageorgiou, E. Parau, Benjamin-Ono Kadomtsev-Petviashvili's models in interfacial electro-hydrodynamics, Eur. J. Mech. B Fluids 65 (2017) 459-463.

[26] W. Choi, R. Camassa, Exact evolution equations for surface waves, J. Eng. Mech. 125 (7) (1999) 756-760.

[27] P.A. Milewski, J.-M. Vanden-Broeck, Z. Wang, Dynamics of steep twodimensional gravity-capillary solitary waves, J. Fluid Mech. 664 (2010) 466-477.
[28] Y.A. Li, J.M. Hyman, W. Choi, A numerical study of the exact evolution equations for surface waves in water of finite depth, Stud. Appl. Math. 113 (3) (2004) 303-324.

[29] T. Gao, J.-M. Vanden-Broeck, Z. Wang, Numerical computations of twodimensional flexural-gravity solitary waves on water of arbitrary depth, IMA J. Appl. Math. 83 (3) (2018) 436-450.

[30] T. Gao, J.-M. Vanden-Broeck, Numerical studies of two-dimensional hydroelastic periodic and generalised solitary waves, Phys. Fluids 26 (8) (2014) 087101. 